\title{
MMseqs2: sensitive protein sequence searching for the analysis of massive data sets
}

\author{
Martin Steinegger ${ }^{1,2}$ G Johannes Söding ${ }^{1}$ \\ ${ }^{1}$ Quantitative and Computational Biology group, Max-Planck Institute for Biophysical Chemistry, Am Fassberg 11, 370ry Göttingen, \\ Germany; ${ }^{2}$ Department for Bioinformatics and Computational Biology, Technische Universität München, 85748 Garching, Germany
}

e-mail: johannes.soeding@mpibpc.mpg.de

Sequencing costs have dropped much faster than Moore's law in the past decade, and sensitive sequence searching has become the main bottleneck in the analysis of large 5 metagenomic datasets. We therefore developed the open-source software MMseqs2 60 (mmseqs.org), which improves on current search tools over the full range of speed-sensitivity trade-off, achieving 10 sensitivities better than PSI-BLAST at more than 400 times its speed.

Owed to the drop in sequencing costs by four orders of magnitude since 2007, many large-scale metagenomic projects each 15 producing terabytes of sequences are being performed, with applications in medical, biotechnological, microbiological, and

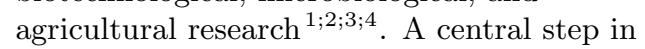
the computational analysis is the annotation 20 of open reading frames by searching for similar sequences in the databases from which to infer their functions. In metagenomics, computational costs now dominate sequencing $\operatorname{costs} \frac{\sqrt{5 / 617}}{}$ and protein 25 searches typically consume $>90 \%$ of computational resources ${ }^{7}$, even though the sensitive but slow BLAST ${ }^{8]}$ has mostly been

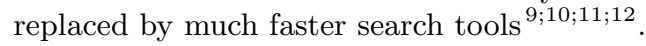
But the gains in speed are paid by lowered 30 sensitivity. Because many species found in metagenomics and metatranscriptomics studies are not closely related to any organism with a well-annotated genome, the fraction of unannotatable sequences is often 35 as high as $65 \%$ to $90 \% \frac{13 \mid 2}{12}$, and the widening gap between sequencing and computational costs quickly aggravates this problem.

To address this challenge, we developed the parallelized, open-source software suite 40 MMseqs2. Compared to its predecessor MMseqs $\frac{14}{14}$, it is much more sensitive, supports iterative profile-to-sequence and sequence-to-profile searches and offers much enhanced functionality (Supplementary ${ }_{45}$ Table S I).

MMseqs2 searching is composed of three stages (Fig. 1a): a short word (" $k$-mer") match stage, vectorized ungapped alignment, and gapped (Smith-Waterman) alignment.

50 The first stage is crucial for the improved performance. For a given query sequence, it 105 finds all target sequences that have two consecutive similar- $k$-mer matches on the same diagonal (Fig. 1 b). Consecutive $k$-mer
55 matches often lie on the same diagonal for homologous sequences (if no alignment gap occurs between them) but are unlikely to do so by chance. Whereas most fast tools detect only exact $k$-mer matches $\frac{9|10| 11 \mid 12}{}$, MMseqs2, 60 like MMseqs and BLAST, finds $k$-mer matches between similar $k$-mers. This similar- $k$-mer matching allows MMseqs2 to use a large word size $k=7$ without loosing sensitivity, by generating a large number of 65 similar $k$-mers, $\sim 600$ to 60000 per query $k$-mer depending on the similarity setting (Fig. 1 b, orange frame). Importantly, its innermost loop 4 needs only a few CPU clock cycles per $k$-mer match using a trick to 70 eliminate random memory access (last line in magenta frame, Supplementary Fig. S1).

The critical insight was to follow our credo "information is power" by combing the double-match criterion with making $k$-mers

75 as long as possible, which required finding similar and not just exact $k$-mers. This effectively bases our decision on up to $2 \times 7=14$ residues instead of just $2 \times 3$ in BLAST or 12 letters on a size-11 alphabet in 80 DIAMOND.

MMseqs2 is parallelized on three levels: time-critical parts are manually vectorized, queries can be distributed to multiple cores, and the target database can be split into 85 chunks distributed to multiple servers.

Because MMseqs2 needs no random memory 140 access in its innermost loop, its runtime scales almost inversely with the number of cores used (Supplementary Fig. S2).

90 MMseqs2 requires $13.4 \mathrm{~GB}$ plus $7 \mathrm{~B}$ per amino acid to store the database in memory, 14 or $80 \mathrm{~GB}$ for $30.3 \mathrm{M}$ sequences of length 342 . Large databases can be searched with limited main memory by splitting the database 95 among servers, at very moderate loss of speed (Supplementary Fig. S3).

We developed a benchmark with full-length sequences containing disordered, low-complexity and repeat regions, because 100 these regions are known to cause false-positive matches, particularly in iterative profile searches. We annotated UniProt sequences with structural domain annotations from SCOP ${ }^{15}, 6370$ of which 105 were designated as query sequences and $3.4 \mathrm{M}$ as database sequences. We also added 160 $27 \mathrm{M}$ reversed UniProt sequences, thereby preserving low complexity and repeat structure $^{\sqrt{16}}$. The unmatched parts of query 110 sequences were scrambled in a way that conserved the local amino acid composition. A benchmark using only unscrambled sequences gives similar results

(Supplementary Figs. S4, S5, S6, S7). 115 We defined true positive matches to have annotated SCOP domains from the same SCOP family, false positives match a reversed sequence or a sequence with a SCOP domain from a different fold. Other 120 cases are ignored.

Figure 2a shows the cumulative distribution of search sensitivities.

Sensitivity for a single search is measured by the area under the curve (AUC) before the 125 first false positive match, i.e., the fraction of true positive matches found with better $E$-value than the first false positive match. MMseqs2-sensitive reaches BLAST's sensitivity while being 36 times faster.

130 Interestingly, MMseqs2 is as sensitive as the exact Smith-Waterman aligner SWIPE ${ }^{17}$, compensating some unavoidable loss of sensitivity due to its heuristic prefilters by effectively suppressing false positive matches 135 between locally biased segments (Fig. 2d, Supplementary Fig. S4). This is achieved by correcting the scores of regions with biased amino acid composition or repeats, masking such regions in the $k$-mer index using TANTAN ${ }^{18}$, and reducing homologous overextension of alignments $\frac{19}{19}$ with a small negative score offset (Fig. 2d,

Supplementary Fig. S7). All tools except MMseqs2 and LAST reported far too 5 optimistic $E$-values (Supplementary Fig. S8). For example in the 6370 searches DIAMOND reported 69211 false positive matches with E-values below $10^{-3}$ (versus 0.637 expected) in $5 \%$ of the searches (versus ${ }_{150} 0.1 \%$ expected), while MMseqs 2 produced 54 false positive matches with $E<10^{-3}$ in only $0.1 \%$ of the searches (Supplemental Table S1). In automatic functional annotation pipelines, such unreliable $E$-values will lead 155 to an increased fraction of false annotations.

In a comparison of AUC sensitivity and speed (Fig. 2b), MMseqs2 with four sensitivity settings (red) shows the best combination of speed and sensitivity over the 160 entire range of sensitivities. Similar results were obtained with a benchmark using unscrambled or single-domain query 
bioRxiv preprint doi: https://doi.org/10.1101/079681; this version posted June 7, 2017. The copyright holder for this preprint (which was not certified by peer review) is the author/funder, who has granted bioRxiv a license to display the preprint in perpetuity. It is made available under aCC-BY-NC-ND 4.0 International license.

Figure 1: MMseqs2 searching in a nutshell. (a) Three increasingly sensitive search stages find similar sequences in the target database. (b) The short word (" $k$-mer") match stage detects consecutive inexact $k$-mer matches occurring on the same diagonal. The diagonal of a $k$-mer match is the difference between the positions of the two similar $k$-mers in the query and in the target sequence. The pre-computed index table for the target database (blue frame) contains for each possible $k$-mer the list of the target sequences and positions where the $k$-mer occurs (green frame). Query sequences/profiles are processed one by one (loop 1). For each overlapping, spaced query $k$-mer (loop 2), a list of all similar $k$-mers is generated (orange frame). The similarity threshold determines the list length and sets the trade-off between speed and sensitivity. For each similar $k$-mer (loop 3) we look up the list of sequences and positions where it occurs (green frame). In loop 4 we detect consecutive double matches on the same diagonals (magenta and black frames).

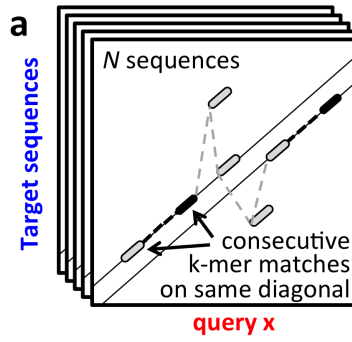

Fast $k$-mer match stage (a)

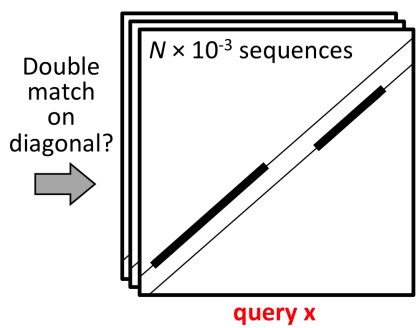

Vectorized ungapped alignment
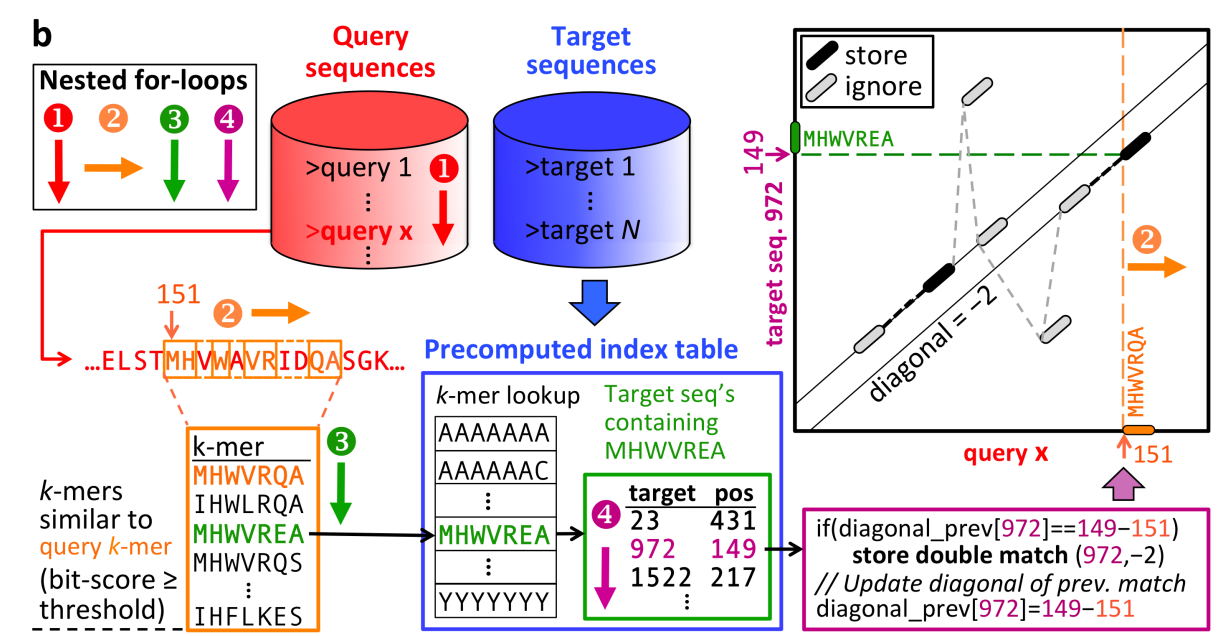

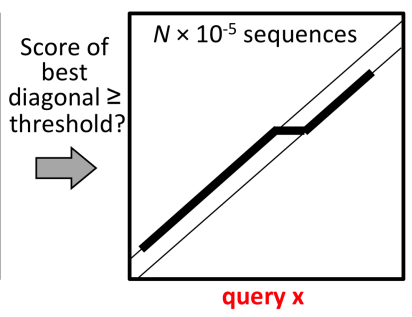

Vectorized gapped alignment sequences (Supplementary Figs. S4, S5, 195 for $67 \%$ of the 40.2 M OM-RGC genes 1 . We S6, S7, S9, S10).

165 Searches with sequence profiles are generally much more sensitive than simple sequence searches, because profiles contain detailed, family-specific preferences for each amino acid at each position. We compared 170 MMseqs2 to PSI-BLAST (Fig. 2b) using two to four iterations of profile searches through the target database. As expected, MMseqs2 profile searches are much faster and more sensitive than BLAST sequence 175 searches. But MMseqs2 is also considerably more sensitive than PSI-BLAST, despite being 433 times faster at 3 iterations. This is partly owed to its effective suppression of high-scoring false positives and more

180 accurate $E$-values (Fig. 2d,

Supplementary Fig. S7).

The MMseqs2 suite offers workflows for various standard use cases of sequence and profile searching and clustering of huge 185 sequence datasets and includes many utility scripts. We illustrate its power with three example applications.

In the first example, we tested MMseqs2 for annotating proteins in the Ocean 190 Microbiome Reference Gene Catalog (OM-RGC) $)^{1}$. The speed and quality bottleneck is the search through the eggNOGv3 database 20 . The BLAST search with $E$-value cutoff 0.01 produced matches replaced BLAST with three MMseqs2 searches of increasing sensitivity

(Supplementary Fig. S11). The first MMseqs2 search in fast mode detected 200 matches for $59.3 \%$ of genes at $E \leq 0.1$. ( $E \leq 0.1$ corresponds to the same false discovery rate as $E \leq 0.01$ in BLAST, Fig. 2d). The sequences without matches were searched with default sensitivity, and $17.5 \%$ 205 had a significant match. The last search in sensitive search mode found matches for $8.3 \%$ of the remaining sequences. In total we obtained at least one match for $69 \%$

sequences in OM-RGC, $3 \%$ more than

210 BLAST, in $1 \%$ of the time (1520 vs. 162952

CPU hours; Shini Sunagawa, personal communication).

In the second example, we sought to annotate the remaining $12.3 \mathrm{M}$ unannotated 215 sequences using profile searches. We merged the UniProt database with the OM-RGC sequences and clustered this set with MMseqs2 at 50\% sequence identity cut-off. We built a sequence profile for each 220 remaining OM-RGC sequence by searching through this clustered database and accepting all matches with $E \leq 0.001$. With the resulting sequence profiles we searched through eggNOG, and $3.5 \mathrm{M}(28.3 \%)$ profiles 225 obtained at least one match with $E<0.1$. This increased the fraction of OM-RGC sequences with significant eggNOG matches to $78 \%$ with an additional CPU time of 900 hours. In summary, MMseqs 2 matched $78 \%$ 230 sequences to eggNOG in only $1.5 \%$ of the $\mathrm{CPU}$ time that BLAST needed to find matches for $67 \%$ of the OM-RGC sequences ${ }^{1}$.

In the third example, we annotated a non-redundant set of 1.1 billion hypothetical 235 proteins sequences with Pfam ${ }^{21}$ domains. We predicted these sequences of average length 134 in $\sim 2200$

metagenome/metatranscriptome datasets 22 . Each sequence was searched through 16479

240 Pfam31.0 sequence profiles held in $16 \mathrm{~GB}$ of memory of a single $2 \times 14$-core server using sensitivity setting $-\mathrm{s}$ 5. Supplementary Fig. S12 explains the adaptations to the k-mer prefilter and search workflow. The 245 entire search took 8.3 hours, or $0.76 \mathrm{~ms}$ per query sequence per core and resulted in $370 \mathrm{M}$ domain annotations with $E$-values below 0.001 . A search of 1100 randomly sampled sequences from the same set with 250 HMMER3 ${ }^{23}$ through Pfam took 10.6 s per seqeunce per core, almost 14000 times longer, and resulted in 514 annotations with $E<0.001$, in comparison to 415 annotations found by MMseq2. A sensitivity setting of 255 -s 7 brings the number of MMseqs2 annotations to 474 at 4000 times the speed of HMMER3.

In summary, MMseqs2 closes the cost and 
bioRxiv preprint doi: https://doi.org/10.1101/079681; this version posted June 7, 2017. The copyright holder for this preprint (which was not certified by peer review) is the author/funder, who has granted bioRxiv a license to display the preprint in perpetuity. It is made available under aCC-BY-NC-ND 4.0 International license.

Figure 2: MMseqs2 pushes the boundaries of sensitivity-speed trade-off. a Cumulative distribution of Area under the curve (AUC) sensitivity for all 6370 searches with UniProt sequences through the database of $30.4 \mathrm{M}$ full-length sequences. Higher curves signify higher sensitivity. Legend: speedup factors relative to BLAST, measerver using a 100 times duplicated query set (637000 sequences). Times to index the database have not been included. MMseqs2 indexing takes 11 minutes for $30.3 \mathrm{M}$ sequences of avg. length 342. b Average AUC sensitivity versus speedup factor relative to BLAST. White numbers in plot symbols: number of search iterations. c Same analysis as in a, for iterative profile searches. d False discovery rates for sequence and profile searches. Colors: as in a (top) and c (bottom). sured on a $2 \times 8$ core 128 GB RAM

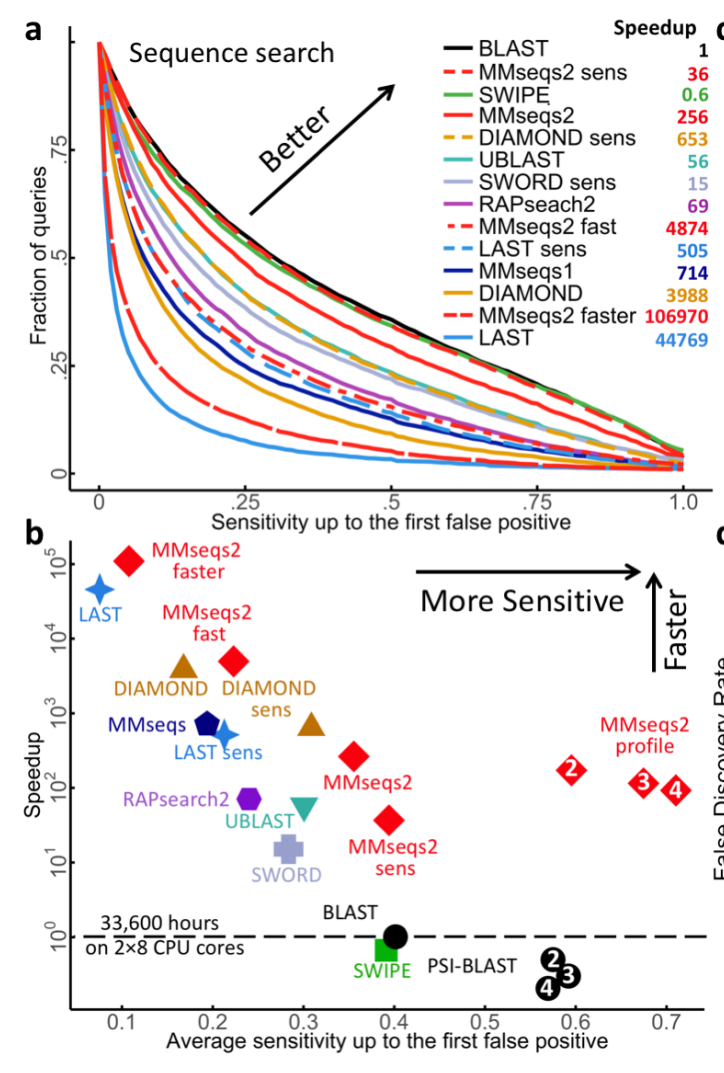

285 AUTHOR CONTRIBUTIONS

performance gap between sequencing and 260 computational analysis of protein sequences. Its sizeable gains in speed and sensitivity should open up new possibilities for analysing large data sets or even the entire genomic and metagenomic protein sequence 265 space at once.

\section{ACKNOWLEDGEMENTS}

We are grateful to Cedric Notredame and Chaok Seok for hosting M.S. at the CRG in Barcelona and at Seoul National University 270 for 12 and 18 months, respectively, and to Burkhard Rost at TU Munich for accepting the formal supervision of his $\mathrm{PhD}$ thesis. We thank Milot Mirdita, Lars van den Driesch and Clovis Galiez for contributing utilities 275 and workflows, and Shini Sunagawa, Martin Frith, Thomas Rattei and our lab for feedback on the manuscript. This work was supported by the European Research Council's Horizon 2020 Framework

280 Programme for Research and Innovation ("Virus-X", project no. 685778) and by the German Federal Ministry for Education and Research (BMBF) (grants e:AtheroSysMed 01ZX1313D, "SysCore" 0316176A).

\section{M.S. developed the software and performed} the data analysis. M.S. and J.S. conceived of and designed the algorithms and benchmarks and wrote the manuscript.

\section{${ }_{290}$ COMPETING FINANCIAL INTERESTS}

The authors declare no competing financial interests.

\section{References}

[1] Sunagawa, S. et al. Science 348, 1261359-1-9 (2015).

[2] Afshinnekoo, E. et al. Cell Systems 1, 72-87 (2015).

[3] Howe, A. C. et al. Proc. Natl. Acad. Sci. U.S.A. 111, 4904-4909 (2014).

[4] Franzosa, E. A. et al. Nat. Rev. Microbiol. 13, 360-372 (2015).

[5] Scholz, M. B. et al. Curr. Opin. Biotechnol. 23, 9-15 (2012).

[6] Desai, N. et al. Curr. Opin. Biotechnol. 23, 72-76 (2012).
[7] Tang, W. et al. Workload characterization for MG-RAST metagenomic data analytics service in the cloud. In IEEE International Conference on Big Data, 56-63 (IEEE, 2014).

[8] Altschul, S. F. et al. Nucleic Acids Res. 25, 3389-3402 (1997).

[9] Edgar, R. C. Bioinformatics 26, 2460-2461 (2010).

[10] Kiełbasa, S. M. et al. Genome Res. 21, 487-493 (2011).

[11] Zhao, Y. et al. Bioinformatics 28, 125-126 (2012).

[12] Buchfink, B. et al. Nature Methods 12, 59-60 (2015).

[13] Hurwitz, B. L. \& Sullivan, M. B. PLoS One 8, e57355 (2013).

[14] Hauser, M. et al. Bioinformatics 32, 1323-1330 (2016).

[15] Murzin, A. G. et al. J. Mol. Biol. 247, $536-540$ (1995).

[16] Karplus, K. et al. Bioinformatics 14, 846-856 (1998). 
bioRxiv preprint doi: https://doi.org/10.1101/079681; this version posted June 7, 2017. The copyright holder for this preprint (which was not certified by peer review) is the author/funder, who has granted bioRxiv a license to display the preprint in perpetuity. It is made available under aCC-BY-NC-ND 4.0 International license.

[17] Rognes, T. BMC Bioinformatics 12, $221+(2011)$.

[18] Frith, M. C. Nucleic Acids Res. 39, e23-e23 (2011). URL

http://www.ncbi.nlm.nih.gov/pmc/

articles/PMC3045581/.
[19] Frith, M. C. et al. Nucleic Acids Res. 36, 5863-5871 (2008).

[20] Jensen, L. J. et al. Nucleic Acids Res. 36, D250-D254 (2008).

[21] Finn, R. D. et al. Nucleic Acids Res. 44,
D279-D285 (2016).

[22] Steinegger, M. \& Söding, J. bioRxiv 104034 (2017).

[23] Eddy, S. R. PLoS Comput. Biol. 7, e1002195 (2011). 